\title{
Experimental Hydraulic Analysis of Intake Structure for Cooling Towers Pumps
}

\author{
Gregor Žvab1,* - Gregor Lapuh² \\ 1 Kolektor-Turboinštitut d.o.o., Slovenia \\ 2 Krško Nuclear Power Plant, Slovenia
}

\begin{abstract}
Experimental hydraulic analysis of troubleshooting intake structures for cooling towers (CT) pumps in nuclear power plant are presented in this paper. The formation of the free surface vortex in the pump sump with unfavorable intensity was not acceptable for the safe operation of the cooling towers pump and the cooling system in general. The objective of the present study is to investigate and improve hydraulic flow conditions in intake structure of CT pumping stations within acceptable criteria of the ANSI/HI standard experimentally. For this purpose, the physical hydraulic model with the scale factor 16 was made. Boundary conditions were calculated by dimensional analysis with the Froude number. The vorticity of flow in the suction pipe of the model pumps was measured and free surfaces and submerged vortices were observed. The model tests were made for all operating conditions of pumps. Comparison between the model and prototype showed a good agreement by the formation of an identical free surface vortex in the same area of the sump. In order to prevent the formations of the unfavorable free surface vortex, the geometry of intake structures with the additional elements and obstacles were modified. Measured and observed results for additional tests have been obtained. The optimized geometry of intake structures provided favorable flow conditions by safety factor 1.5 within acceptable criteria of the ANSI/HI standard.
\end{abstract}

Keywords: intake structures, physical hydraulic model, free surface flow, free surface vortices, vertical pump

\footnotetext{
Highlights

- $\quad$ The troubleshooting of formations the free surface vortices in the pump sump with unfavorable intensity was not acceptable for the safe operation of the pump, therefore an experimental hydraulic analysis was carried out to improve the flow conditions of pump intakes in accordance of the ANSI/HI standard.

- Comparison between the model and prototype showed a good agreement by the formation of an identical free surface vortex in the same area of the sump.

- The study confirmed previous research that a strong cross flow near the pump intakes results in the formation of more intense streamlines at the free surface level.

- $\quad$ The undesirable influence of the cross flow was successfully prevented.
}

\section{INTRODUCTION}

The inlet structures are a segment of the pumping system through which a source of fluid is supplied, and should be directed toward the pump intakes as uniformly as possible. Improperly designed intake structures can lead to unfavorable hydraulic disturbances in the flow which adversely affect the pump operation in its entire lifetime [1]. Consequently, it leads to poor pumping efficiency, damage to the vital parts of the pump, or even to failure [2]. In case of failures, additional maintenance work and remediation are required which impair the pump availability and the pump economy [3]. In general, intake structures are divided into clear liquid and intake structures for solid bearing liquid. After that, intake structures are further divided into different shapes of geometry. To prevent unfavorable hydraulic disturbance, additional elements and obstacles are installed into the sump. Unfavorable hydraulic disturbances occur in the form of surface and sub-surface vortices, non-uniform axial velocities of the flow at the pump inlet, and the leak of air and air bubbles into the pump [1].

The beginnings of the experimental research of undesirable hydraulic phenomena in intake structures date to 1950 [4]. Since then, numerous empirical relationships have been obtained between the geometric and hydraulic parameters of the pump, intake structures, and unfavorable hydraulic phenomena in the flow. Recommendations have been obtained through researches and developments in the pump industry from different authors until now. Guidelines for the design of intake structures are compiled in the ANSI/HI standard [4].

From case to case, intake structures require specific design because of different boundary conditions. In general, empirical results, correlations, and different parameter relationships are difficult to apply in the specific case. The ANSI/HI standard recommends the physical model tests for the design of a flow of pump greater than $2.5 \mathrm{~m}^{3} / \mathrm{s}$ and the pump station flow greater than $6.3 \mathrm{~m} 3 / \mathrm{s}$. 
In general, it is recommended that a direction of approaching flow should coincide with the flow direction near the pump intake. Otherwise, the unfavorable cross-flow may appear near the pump [5]. In case of intense cross flow in the pump sump, the streamlines of flow become more intense at the free surface of the liquid. If the pump is not sufficiently submerged, it may lead to the formation of free vortices. During past experimental studies, numerous authors focused on the critical submergence of the pump (Scr). Quick [6] was the first to describe the $\mathrm{Scr}$ parameter by formations of free surface vortices. It is the relationship between pump bell diameter $D$ and Froude $(F r)$ number. The effect of Reynolds $(R e)$ number and Weber number $(\mathrm{We})$ was investigated by numerous authors. They concluded that it can be neglected in case of sufficiently large $R e$ and $W e$ numbers. Subsequently, the limit values for $R e$ and $W e$ were recommended in the ANSI/HI standard [4].

The geometry of intake structures and of the pump also has an important influence on unfavorable hydraulic disturbances. For example, pre-swirl near the pump bell is increased if the distance between the pump axis and the back wall or the side wall is increased excessively [7]. The similar results were also obtained by Padmanabhan and Hecker [8] and by Anwar and Amimilett [9] studies. Studies by Quick [6] and Tagamori and Ueda [10] confirmed that reduction of the back wall, side wall, and the bottom floor clearances increases the risk of formation of subsurface vortices which originate from there. For this purpose, various elements and obstacles must be properly installed near the pump intake. In addition, Denny [7] suggested that geometry of the pump bell has a noticeable influence on flow separation and increases the non-uniform velocities in the approach flow. In the case of parallel installation of pumps, it can cause interaction of flows between pumps which are installed too close to the sump. For this purpose, it is necessary to design intermediate walls between pumps to prevent flow interaction. For dissipation water energy, different elements and obstacles are also installed to reduce flow velocities and other undesirable hydraulic phenomena. The velocity of flow can also be reduced with the gradual expansion of side walls in the pump sump.

In recent studies for examining the flow pattern in the approaching flow, acoustic Doppler velocimetry (ADV) was employed [11]. Hwang and Yang [12] experimentally measured approaching flow using ADV and swirl meter and provide valuable information for practical application.
Flow conditions in intake structures can also be predicted with the computational fluid dynamic (CFD). Numerous authors [13] to [16] simulated flow patterns using advanced numerical modeling. They also compared or validated their simulations with measurements taken using particle image velocimetry (PIV). Calculation of free surface flow with formations of vortices usually requires extremely fine meshing and advanced turbulent models with considerations of two-phase flow. However, this is limited by the computational power and storage [4].

The experimental study deals with the hydraulic analysis in practice. The formation of the free surface vortex in the pump sump with unfavorable intensity was not acceptable for the safe operation of the CT1 pump and cooling system in general. The objective of the present study is to experimentally investigate and improve hydraulic flow conditions in intake structure of CT pumping stations within acceptable criteria of the ANSI/HI standard. For the purpose of the study, detailed knowledge of the flow conditions at the pumping station was needed.

\section{METHODS}

Using the design requirements given in the ANSI/HI standard, the geometry and hydraulic parameters for the intake structure and the pump must be properly selected for satisfactory hydraulic performance. Fig. 1 shows the design criteria for rectangular intake structures for clear liquids in plan and elevation view.
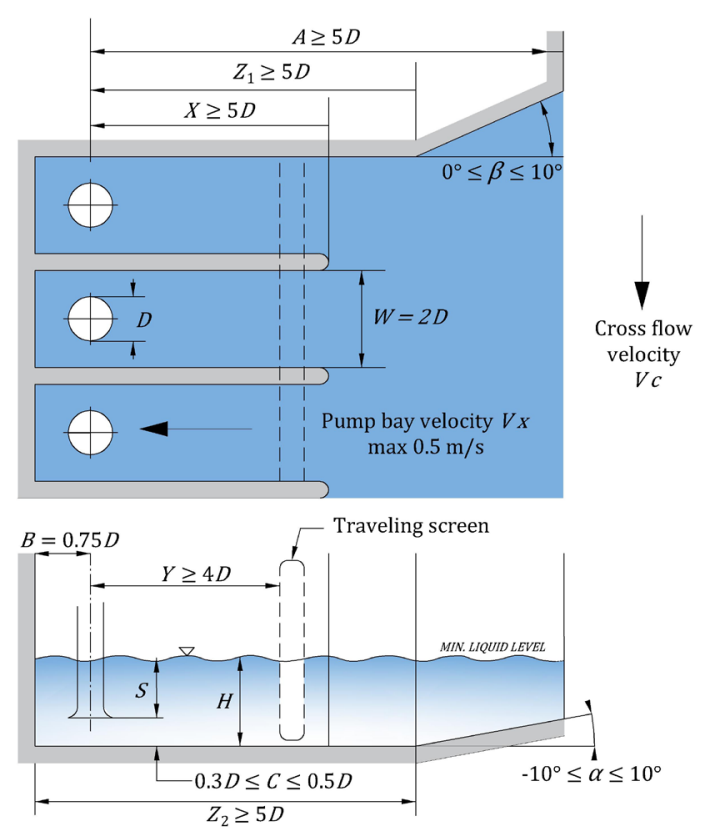

Fig. 1. Design criteria for the rectangular intake structures 
A physical hydraulic model study shall be conducted for rectangular intake structures with one or more of the following features:

- Sump or pump geometry deviates from recommendations of the ANSI/HI standard.

- Non-uniform of non-symmetric approach flow to the pump sump exists.

- The flows of the pumps greater than $2.5 \mathrm{~m}^{3} / \mathrm{s}$ per pump or the total station flow with all the pumps running would be greater than $6.3 \mathrm{~m}^{3} / \mathrm{s}$.

- The cross-flow velocity $(V c)$ in the sump exceeds $50 \%$ of axial velocity in the pump intake.

- Traveling or static screen is mounted in the sump.

\subsection{Physical Hydraulic Modeling of Free Surface Flow}

The mathematical models of the flow of real liquid can be solved by simplified models alone. Therefore, the solutions for many engineering problems are achieved through the use of a combination of theoretically and experimentally obtained data [17]. In this chapter, we consider dimensional analysis, similitude and experimental modeling of free surface flow, as well as measuring techniques in acceptance to the ANSI/ HI standard.

A model is a representation of a physical system that may be used to predict the behavior of the system in some desired respect. The physical system for which the predictions are to be made is called the prototype [17]. The theory of models can be readily developed by using the Buckingham theorem, which is the basis of the dimensional analysis. The model is accurate when all the dimensionless numbers that affect the physical phenomenon are the same on the prototype. In other words, we have to maintain geometric, dynamic, and kinematic similarity.

Flows in canals, rivers, basins, as well as flows around ships, are examples of flow phenomena involving a free surface flow. As shown in Eq. (1), dimensionless groups are affected by the phenomena. The first group $L_{i} / L$ indicates the scale factor of the model. The next group $\varepsilon / \mathrm{L}$ indicates the relative roughness, and the last groups indicate Reynolds $(R e)$, Froude $(F r)$ and Weber number $(W e)$. The influence of viscous effects is defined by the $R e, F r$ representing the ratio of inertial to gravitational forces, while We represents surface tension effect.

$$
\begin{gathered}
\Pi_{i}=\varphi\left(\frac{L_{i}}{L}, \frac{\varepsilon}{L}, R e, F r, W e\right), \\
F r=\frac{V}{\sqrt{g \cdot D}},
\end{gathered}
$$

$$
\begin{gathered}
R e=\frac{V \cdot D}{v}, \\
W e=\frac{\rho \cdot V^{2} \cdot D}{\sigma} .
\end{gathered}
$$

In practice, we must not change the kinematic viscosity and surface tension of the water drastically, so that the models involving free-surface flow are usually distorted. Fortunately, in many problems involving free-surface flows, both surface tension and viscous effects are small and, consequently, the similarity of $W e$ and Re number is not required. Therefore, in free surface flow, $F r$ becomes an important similarity parameter [17] and [18].

$$
F r_{M}=F r_{P} .
$$

By use of Eq. (6) which is derived from dimensional analysis, it may be shown that a given vortex type is a function of various dimensionless parameters. Where $S / D$ is the ratio between submergence of the pump and pump bell diameter, $L_{i} / L$ is a scale factor between model and prototype, $N_{T}$ is circulation number of approach flow and the last one is Froude number.

$$
\Pi_{i}=\varphi\left(\frac{S}{D}, \frac{L_{i}}{L}, N_{T}, F r\right) .
$$

For example, if a scale factor is constant, the equation is simplified with Eq. (7) where terms $F r$ and $S / D$ describe a family of curves. Each one represents different values of vortex strength. There are many equations in the literature with a different relationship between $\mathrm{Scr}$ and $F r$. The ANSI/HI standard uses Eq. (8) by Hecker [4].

$$
\begin{gathered}
\Pi_{i}=\varphi\left(\frac{S}{D}, F r\right), \\
S_{C R}=D(1+2.3 \cdot F r) .
\end{gathered}
$$

\subsection{Physical Model Study Scope}

The objective of the model study is not to investigate flow patterns but to ensure that final sump or piping design generate favorable flow conditions at the inlet to the pump. For studying the potential formation of vortices, it is important to select a reasonably large geometric scale to minimize viscous and surface tension scale effect. The model shall also be large enough to allow visual observations of flow pattern, accurate measurements of swirl and velocity distribution. Selection of the model boundary is also 
extremely important for a proper simulation of flow patterns at the pump. As the approach flow nonuniformities contribute significantly to the undesirable hydraulic phenomena, a sufficient area of the approach geometry has to be modeled, including any channel transition or expansions, obstacles, gates, and any significant cross-flow past the intakes [4].

\subsubsection{Observation of Pump Inlet Disturbances}

The outflow from each simulated pump shall be measured with flow meters. The accuracy of the flow measurements shall be within $\pm 2 \%$ of the actual flow rate. Liquid surface elevation shall be measured using any type of liquid level indicator with the accuracy of at least $3 \mathrm{~mm}$.

To elevate a vortex strength scale, which varies from a surface swirl to an air core vortex, is shown in Fig. 2. Vortices are usually unsteady and, hence, the vortex type shall be estimated through short time interval and determined with an average type during the observation. Subsurface vortices which are determined by the same procedure usually originate at the sump floor and walls and may be visible only when the dye is injected near the vortex core [4].

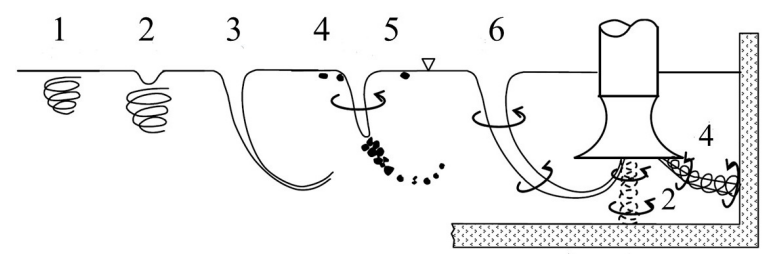

Fig. 2. Classification of free surface and subsurface vortices

\subsubsection{Measuring Swirl in the Pump Suction Pipe}

The intensity of flow rotation shall be measured using a swirl meter (see Fig. 3). It shall consist of a straightvanned propeller with four vanes mounted on a shaft with low-friction bearings, installed in the suction pipe and dimensioned in acceptance to the ANSI/HI standard.

The revolution per unit time of the swirl meter is used to calculate a swirl angle $\theta$ by Eq. (9). Where $d$ is a minimum diameter, $u$ is averaged axial velocity in pump suction pipe, and $n$ is a number of the revolution per second of the swirl meter [4].

$$
\theta=\tan ^{-1}\left(\frac{\pi \cdot d \cdot n}{u}\right) .
$$

In the standard measurement techniques, the measurement of cross-sectional velocity profiles of the approach flow in pump suction pipe is also included. For the purpose of this study, the velocity profiles in pump sump were only measured (see Figs. 10 and 11). The measurement of velocity profiles in pump suction pipe in this study was not included.

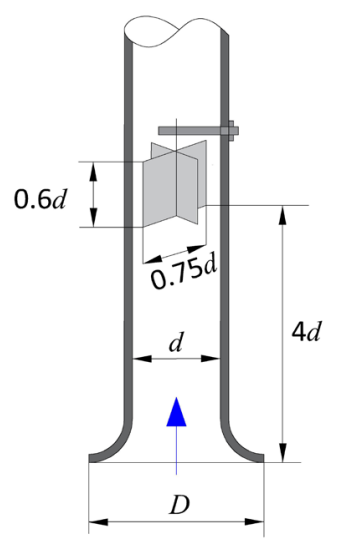

Fig. 3. Typical swirl meter

\section{EXPERIMENTAL}

\subsection{Analysis of CT Pumping System}

The tertiary-cooling system in the Nuclear power plant (see Fig. 4) consists of the condenser, the circulation water (CW) pumping system, the cooling tower (CT) pumping system, the cooling towers, channels, and piping.

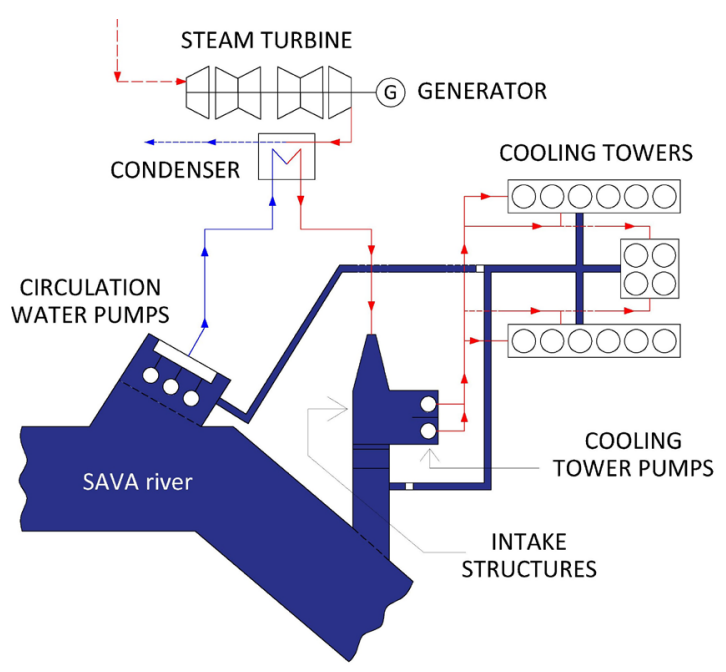

Fig. 4. Scheme of the cooling system in the Nuclear Power Plant in Krško

The cooling system is designed to cool the condenser and dissipate the steam heat that cannot be usefully employed to produce electricity. The 
CW pumps force water from the Sava river into the condenser and back into the river. As it passes through the condenser, the river water heats up because it absorbs heat from the used steam. Since heating the water of the Sava results in the thermal pollution of the river, administrative regulations specify the permitted increase in temperature and the percentage of the river's flow that can be diverted for power plant cooling. In the case of unfavorable meteorological conditions, the cooling towers are used [19].

When only half blocks of the CT operate, only the CT1 pump operates at the CT pumping system. During the summertime, all blocks of the CT usually operate. In this case, both of the CT pumps operate in parallel. The pumps have the same design point with the flow rate of $8.5 \mathrm{~m} 3 / \mathrm{s}$, the head $25 \mathrm{~m}$, and the rotation speed of $427 \mathrm{rpm}$. The open impeller is semi-axial with specific speed $(n q=108)$. The cooling water is pumping through the pipeline with the diameter 1800 $\mathrm{mm}$ to the CT.

The rectangular intake structure on the CT pumping system in Fig. 5 consists of an inlet channel, an extension, an intake sump, and a dam. The pumps are divided by an intermediate wall in the sump. The heated water approaches from the condenser through the inlet channel into the intake structure extension where it decelerates the pump sump. During operation of cooling towers, CT pumps pump the required flow of water in the cooling tower. The remaining heated flow of water flows through the dam to the Sava river.

The formation of the free surface vortex in the pump sump with the type 6 (Fig. 5b) was not acceptable for the safe operation of the pump CT1 and the cooling system in general. The intake structure was investigated with the recommendations of the ANSI/ HI standard. The investigation shows some deficiency which was not taken into the initial design. There, it was found that velocity of cross-flow $V c$ is too high, the width $W$ of the intake channel is too wide, and the distance $B$ and the distance $X$ of the intermediate wall are shorter than recommended.

\subsection{Design of Physical Hydraulic Model}

\subsubsection{Physical Hydraulic Model}

Physical hydraulic model (see Fig. 6) was made in the laboratory for turbomachines at the Kolektor Turboinstitut. It contains a model, a small closedcircuit pumping system with the regulation system and the measuring system. The total flow rate regulation was set with a variable frequency of the pump. The flow rate through the model pumps was regulated by valves on the suction pipes. The intake flow rate was measured by the orifice plate $D / d=90 / 120$ and the pressure transducer. The flow rate over the dam was measured by an electromagnetic flow meter. So the difference between each flow rate means the flow through the model pumps. At the recommended level in the suction pipe of the model pump, standard swirl meter was installed (see Fig. 7c). simultaneously. The water level near the pump was measured by the meter with the accuracy of $2 \mathrm{~mm}$. The errors of flow measurement instruments are presented in Table 1, where $e_{1}$ is a systematic error and $e_{2}$ is a random error.

Table 1. Errors of instruments

\begin{tabular}{lll}
\hline Instrument & $e_{1}$ & $e_{2}$ \\
\hline Pressure transducer/orifice plate & 0.1 & 0.6 \\
\hline Electromagnetic flow meter & 0.5 & 0.2 \\
\hline
\end{tabular}

The investigation of vortices was carried out by observation over the transparent walls around the
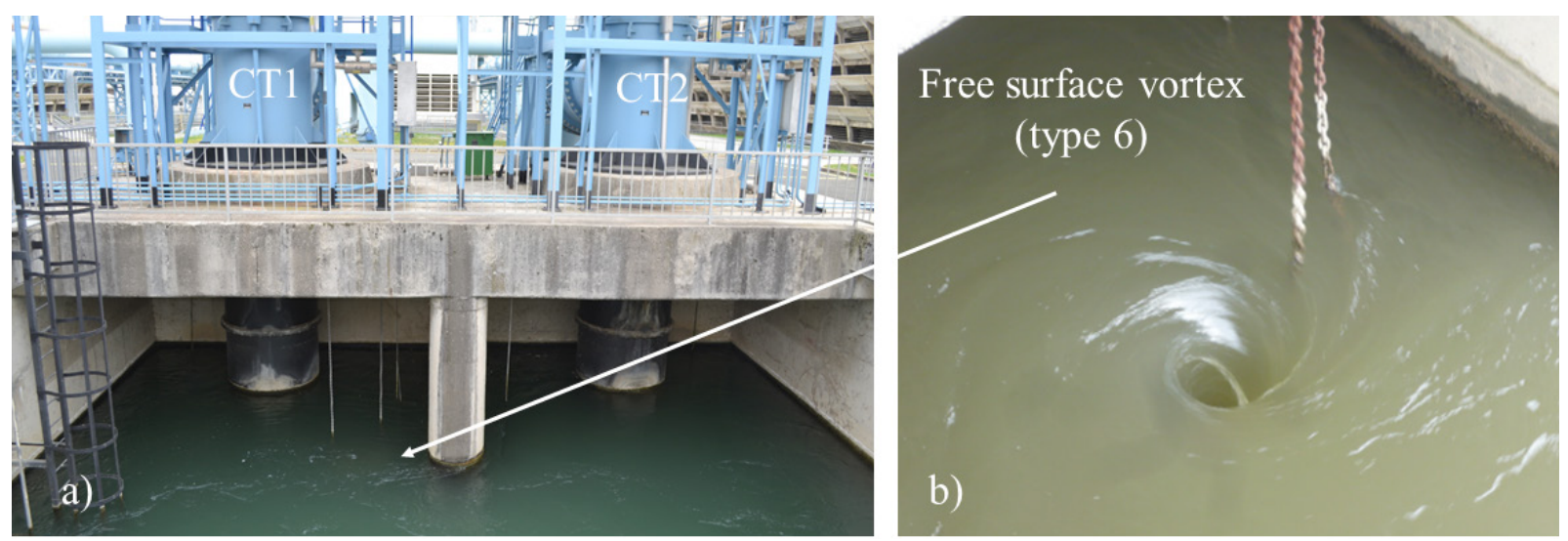

Fig. 5. a) CT pumps with intake structures, b) free surface vortex (type 6) [20] 


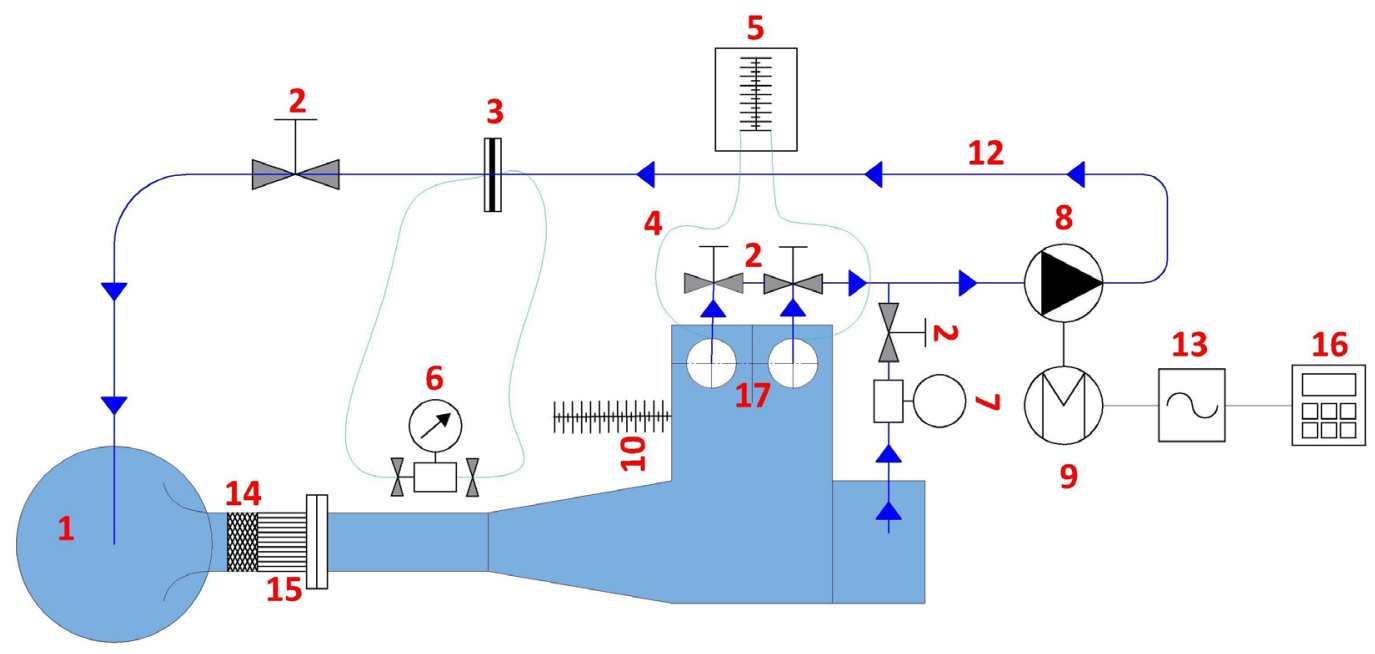

Fig. 6. Test rig of hydraulic model; (1) surge tank, (2) regulating valves, (3) orifice plate, (4) Pitot tube, (5) static head, (6) pressure transducer, (7) electromagnetic flow meter (8) pump, (9) motor, (10) liquid level meter, (11) suction pipe, (12) pressure pipe, (13) frequency converter, (14) mesh, (15) honeycomb tubes, (16) control unit, (17) model pumps
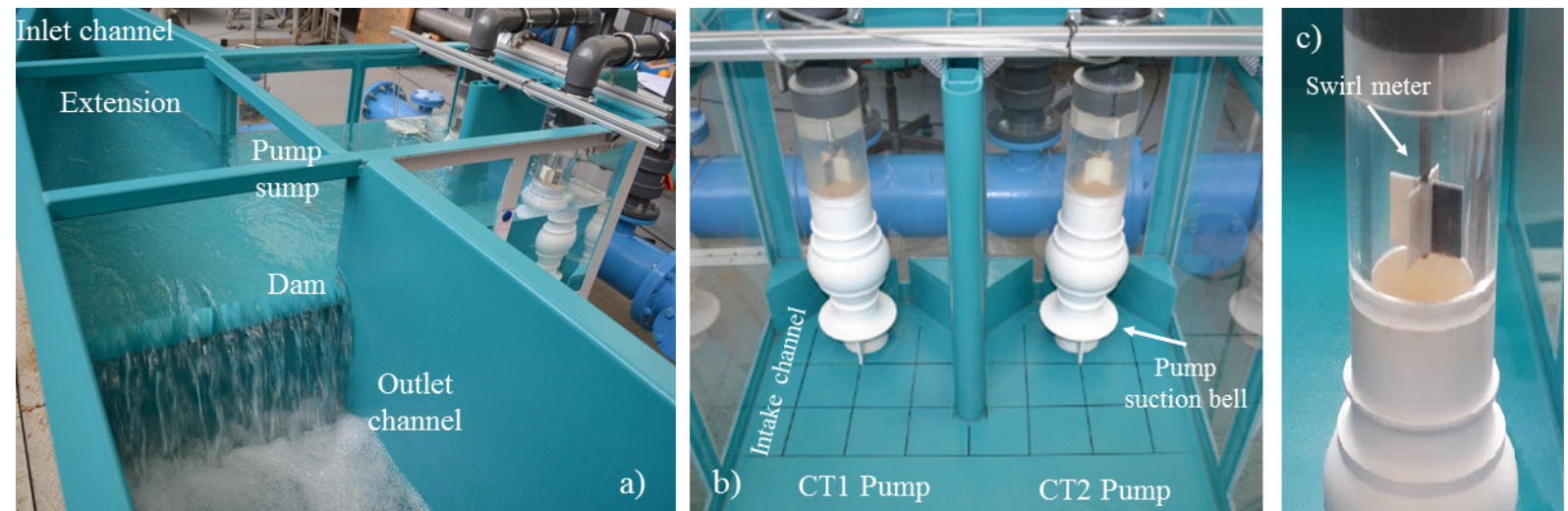

Fig. 7. Physical hydraulic model in the laboratory; a) pump sump with the dam, b) model pumps with the intermediate wall, c) swirl meter [20]

sump. The vortices were documented with a photo and a camera. Subsurface vortices were observed by the injected dye near the pump intake (Fig. 8c).

\subsubsection{Similarity}

A model was designed with the scale factor 16. Hydraulic geometry included an intake channel, an extension, a sump, a model pump suction pipes, and a dam (see Fig. 7a). It was assumed that the outflow channel of the CT pumping system did not have any hydraulic influence on flow condition near the pumps. The walls and floor of the physical model are built with sheet metal and the model pumps are made by 3D print technology (see Fig. 7b). The influence of impeller on flow patterns in the pump intake was neglected in accordance with the ANSI/HI standard. The uniform velocity profile in the inlet channel was simulated by installed meshes and small pipes into the inlet channel. The measuring of depression at the freesurface level was neglected.

\subsubsection{Calibration of the Model}

All the hydraulic dimensions of the model are reduced with the same scale factor. Therefore, geometric similarities were satisfied. The kinematic similarity was satisfied by the similar flows between the model and the prototype for all operating conditions, calculated with Eq. (5). Dynamic similarities were satisfied with the similarity of $F r$. The conditions for the influence of viscous and surface tension were satisfied as well by Eqs. (10) and (11).

$$
\begin{gathered}
R e_{M} \geq 6 \cdot 10^{-4} \\
W e_{M} \geq 240
\end{gathered}
$$




\subsubsection{Measurement Uncertainty}

The measurement uncertainty of filed measurement was neglected. The measurement uncertainty of the intake flow rate $e_{Q z}$ is calculated by Eq. (10), where $e_{z 1}$ is a systematic error of differential pressure transducer, $e_{z 2}$ is a random error of flow through the orifice plate. The measurement uncertainty of the flow rate over the dam $e_{Q d}$ is calculated by Eq. (11) where $e_{d 1}$ is a systematic error of flow meter; $e_{d 2}$ is a random error of flow through the orifice plate. The measurement uncertainty of the total flow rate $e_{Q}$ calculated by Eq. (12) is a geometric sum of measurement uncertainty of $e_{Q \mathrm{z}}$ and $e_{Q d}$.

$$
\begin{gathered}
e_{Q z}=\sqrt{e_{z 1}^{2}+e_{z 2}^{2}}=\sqrt{0.1^{2}+0.6^{2}}=0.7 \%, \\
e_{Q d}=\sqrt{e_{d 1}^{2}+e_{d 2}^{2}}=\sqrt{0.5^{2}+0.2^{2}}=0.5 \%, \\
e_{Q}=\sqrt{e_{Q z}^{2}+e_{Q d}^{2}}=0.86 \%, \quad 0.86 \%<2 \% .
\end{gathered}
$$

\section{RESULTS}

Model tests were carried out in accordance with the ANSI/HI standard. The observations of the undesirable hydraulic phenomena were investigated for all the operating conditions. In addition, the tests were divided on the existed and planned state. For the planned state a higher level of the dam is designed. Hence, the water level in pump sum will be higher. Despite favorable effect on the formation of surface vortices, it is necessary to investigate the flow conditions in both cases.

The results of model tests are given in two parts. In the first part, the results for the existing and the planned state are given. In the second part, we show the results of the tests with the optimization of flow conditions by the selected remedial measures.

\subsection{The First Part}

The existing state: during the operations condition of the CT1 pump, the formation of surface vortices (type 6) was observed (Fig. 8a). The undesirable hydraulic phenomena near the CT2 pump were not observed for all the operations conditions. Subsurface vortices near the pump intake were not observed for any conducted tests (Fig. 9a). The submergence of the pumps $\mathrm{Scr}$ calculated by Hecker Eq. (8) was satisfied for all the operation conditions (Table 2). The excessive swirl angle was only observed in the CT1 pump during the individual operating (Table 3).

Planned state: during the tests with the higher water level in the intake sump, the formations of surface vortices (type 5) were observed (Fig. 9b). During the operations condition of the pump operated in parallel, free-surface vortices with lover intensity (type 3) were observed. Subsurface vortices were not observed in any tests again. The excessive swirl angle was observed during the operations conditions of the CT1 pump again (Table 3 ).

Table 2. Data of the measured submergence and critical submergence of the model pumps

\begin{tabular}{lcccc}
\hline & \multicolumn{2}{c}{ Existing state } & \multicolumn{2}{c}{ Planned state } \\
\cline { 2 - 5 } Operation & $S[\mathrm{~m}]$ & $S c r[\mathrm{~m}]$ & $S[\mathrm{~m}]$ & $S c r[\mathrm{~m}]$ \\
\hline CT1 & 0.236 & 0.286 & 0.299 & 0.293 \\
\hline CT2 & 0.236 & 0.286 & 0.299 & 0.293 \\
\hline CT1+CT2 & 0.217 & 0.286 & 0.280 & 0.293 \\
\hline
\end{tabular}

Results in Table 2 show lower $S$ for parallel operation conditions because of higher depression on
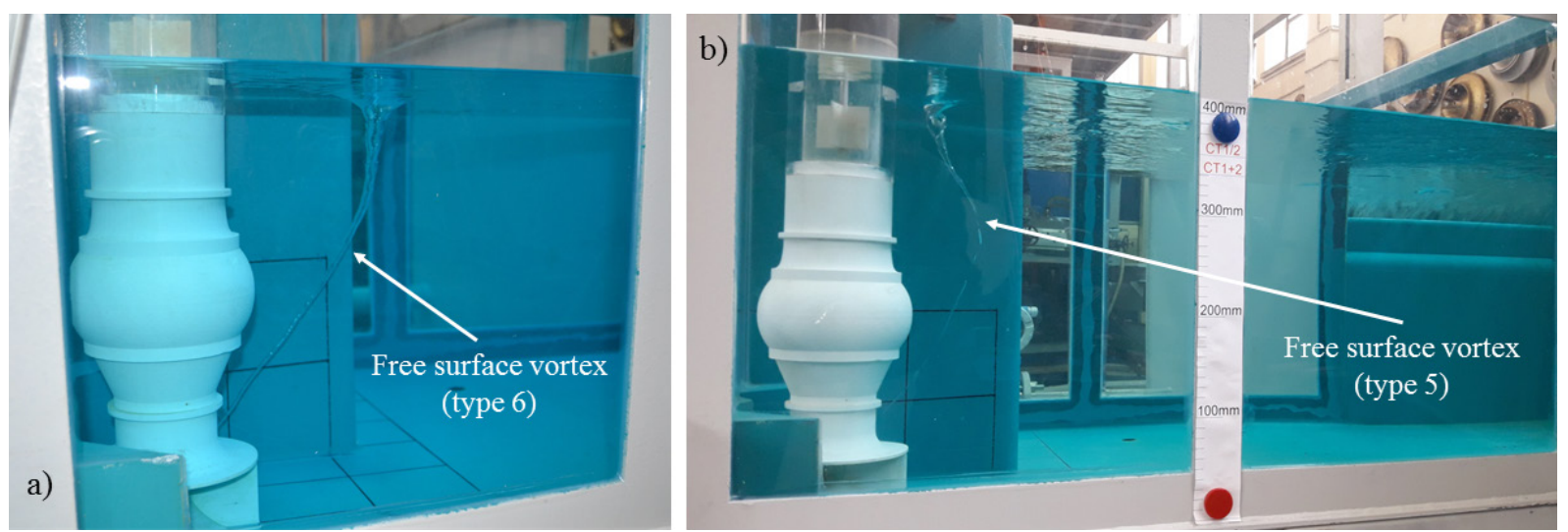

Fig. 8. a) observing the free surface vortices of the existing state and, b) the planned state [20] 

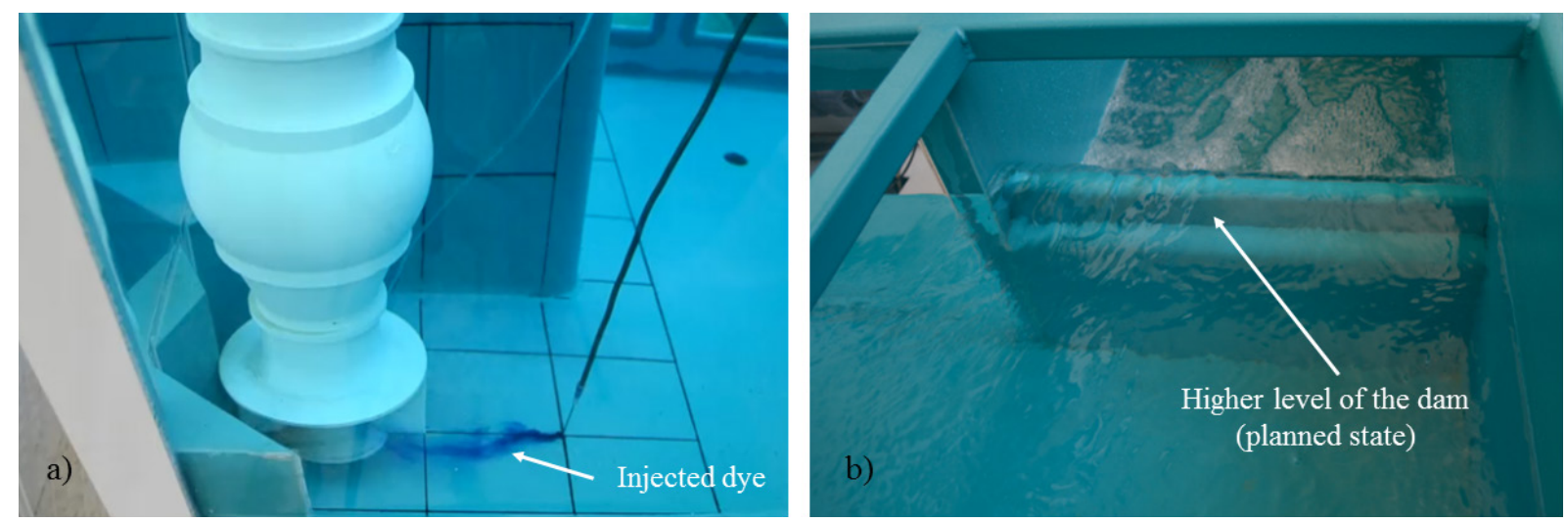

Fig. 9. a) observation of the submerged vortices by injecting dye below the water surface, b) the installed higher level of the dam for the planned state [20]

water surface level near the intake of the pump. For the planned state, a higher $F r$ and $V$ were expected in pump suction pump, so we obtained higher $S c r$ consequently.

Table 3. Calculated swirl angle $\theta$

\begin{tabular}{lcccc}
\hline & \multicolumn{2}{c}{ Existing state } & \multicolumn{2}{c}{ Planned state } \\
\cline { 2 - 5 } Operation & $\mathrm{CT} 1$ & $\mathrm{CT} 2$ & $\mathrm{CT} 1$ & $\mathrm{CT} 2$ \\
\cline { 2 - 5 } & $\theta\left[^{\circ}\right]$ & $\theta\left[^{\circ}\right]$ & $\theta\left[{ }^{\circ}\right]$ & $\theta\left[^{\circ}\right]$ \\
\hline $\mathrm{CT} 1$ & 10.8 & - & 6.3 & - \\
\hline $\mathrm{CT} 2$ & - & 3.31 & - & 2.44 \\
\hline $\mathrm{CT} 1+\mathrm{CT} 2$ & 6.9 & 3.2 & 6.49 & 1.8 \\
\hline
\end{tabular}

Results in Table 3 show correlations with swirl angle $\theta$ and surface vortices. When the surface vortex was formed near the pump intake, the excessive swirl angle $\theta$ has been observed at the same time.
Sketches of the flow: by the comprehensive observations of the flow patterns, it was possible to find out the main reasons for formations of freesurface vortex near the CT1 pump intake. The crossflow velocity $V c$ at the intake of the pump sump has been exceeded [4]. The main flow was oriented over the dam, because of the powerful inertia of the high water velocity $V c$. Velocity profile $V x$ did not coincide with the axis of the CT1 pump due to stagnant and therefore unstable liquid in pump sump. CT1 pump sucked the water only from opposite site of the CT2 pump and, hence, the edge of the intermediate wall formed the flow separation at the free surface around it. Consequently, flow separation formed a coherent air core at the free surface near the CT1 pump intake (see Fig. 10).

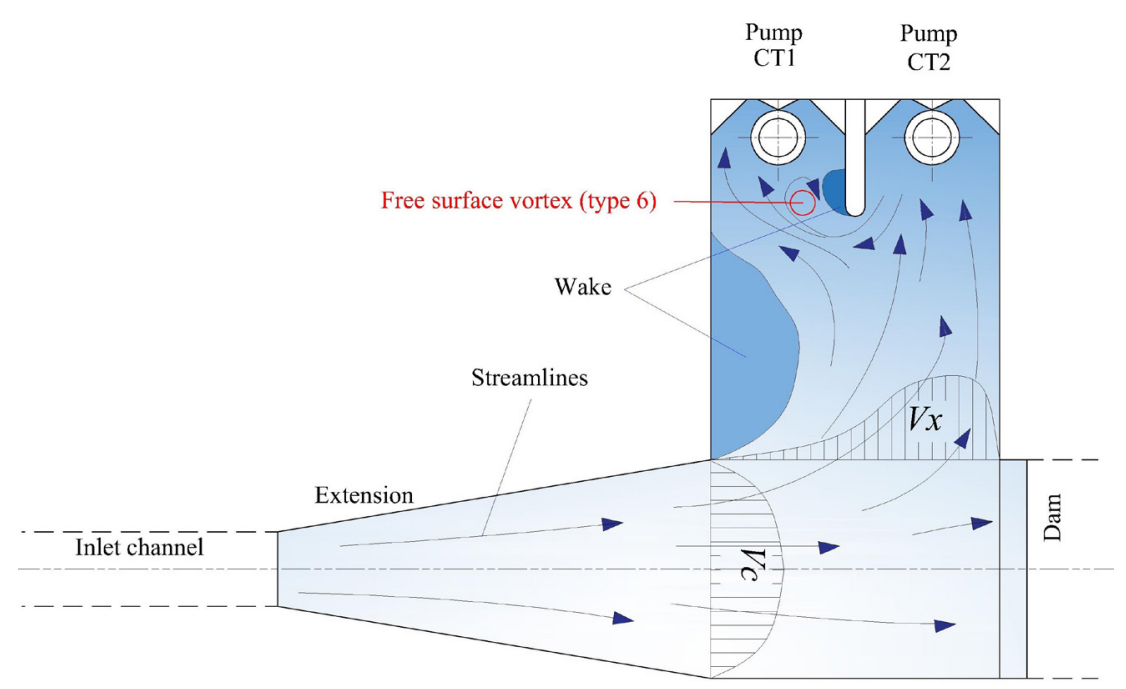

Fig. 10. Sketch of the flow visualization for an individual operation of the CT1 pump 


\subsection{The Second Part}

In order to perform a uniform flow pattern in the approach flow, the appropriate measures with modification geometry of the intake structure have been selected. The measure has been selected on the basis of our experiences and recommendations of the ANSI/HI standard. In the intake sump, the additional inlet wall (Fig. 11a), and the extended intermediate wall (Fig. 11b and c) were mounted. At measure 1 (Fig. 11a), it was necessary to improve the uniformity in the approach flow. Nevertheless, free-surface vortices were still observed. At measure 2 (Fig. 11b), the extended intermediate wall was mounted into the pump sump. The favorable flow conditions in the approach flow were provided.

In order to reduce the construction cost, the same test without an inlet wall was conducted, and the similar results were obtained. The final test was repeated with a higher flow rate by the safety factor 1.5. The optimized geometry of the intake structure with measure 3 (Fig. 11c), provided favorable flow conditions within the acceptable criteria of the ANSI/ HI standard.

\section{DISCUSSION}

Physical hydraulic model of the free surface flow for the intake structures is still an appropriate approach for flow pattern predictions near the pump intake [2] and [4]. In our case, there was a troubleshooting of formations of the free surface vortices (type 6) near the CT1 pump intake, which was not acceptable for safe operation of CT pumping station. Improperly designed intake structure has been analyzed in accordance with the ANSI/HI standard. Intensive free surface vortices may be reduced with increasing depth of submergence $S$ [2] and [4]. During all the tests, the condition $S>$
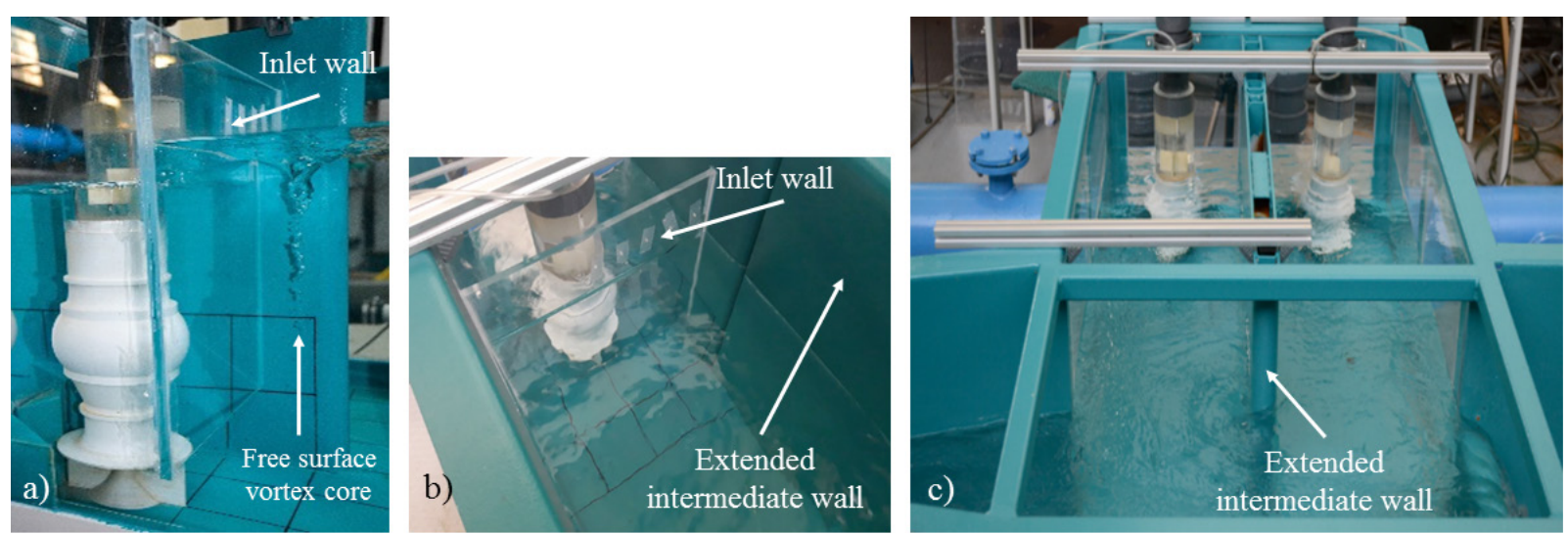

Fig. 11. The selected remedial measures; a) measure 1, b) measure 2, c) measure 3 [20]

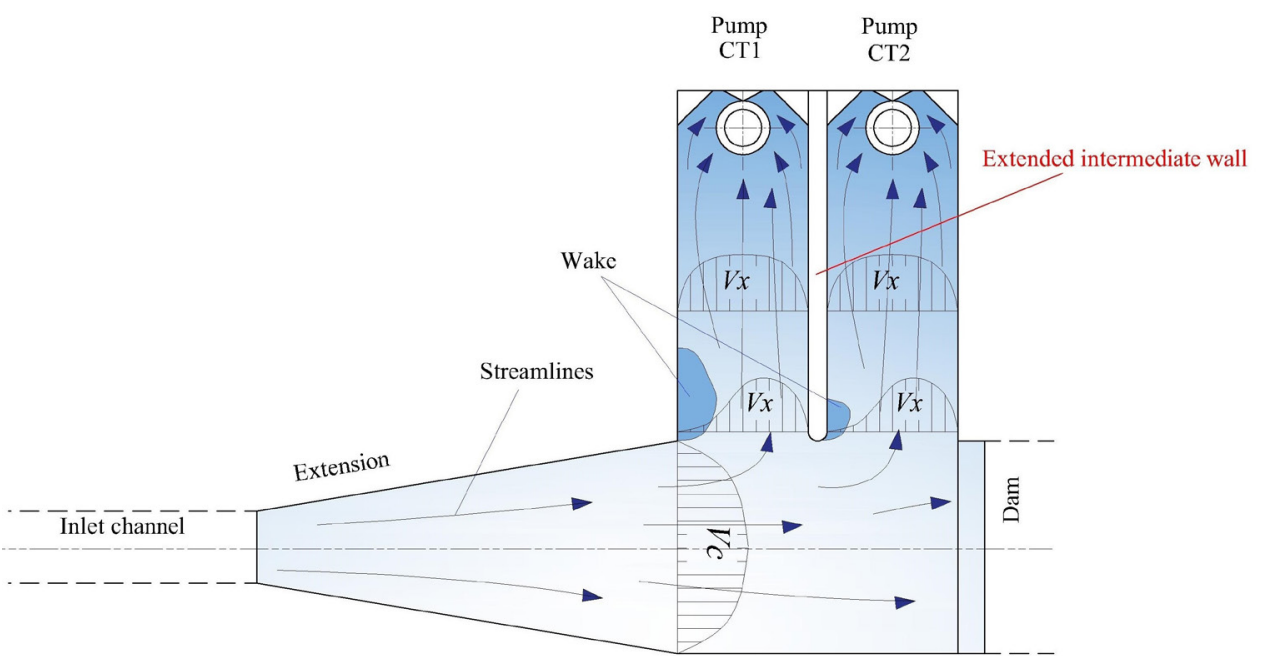

Fig. 12. Sketch of the flow visualization for measure 3 with installed extended intermediate wall (parallel operation of pumps) 
Scr was fulfilled by Eq. (8), Nevertheless, a free surface vortex was forming. However, there are also situations where increasing depth has negligible effect or even increases free surface vortex formations due to stagnant and therefore unstable liquid [4]. As studied by Ansar [5], Ansar and Nakato [11] and Gülich [2], a strong cross flow near the pump intake results in the formations of more intense streamlines at the free surface level. In our case the reason for formation of free surface vortex (type 6) was already explained in the part of the chapter 3 (Sketches of the flow). The undesirable influence of the cross flow was successfully prevented by the installation of the extended intermediate wall between the pumps (see Fig. 12).

\section{CONCLUSIONS}

The study was focused on the design of the intake structure physical model tests to optimize undesirable hydraulic phenomena in free surface flow by the appropriate selection of remedial measures which will be able to apply on the prototype in the CT pumping station in the Nuclear power plant after the investigation. The important outcome of this analysis also confirms a good predictions of flow conditions between prototype and model tests carried out in accordance with the ANSI/HI guidelines. Experiences obtained during analysis can be used in other practical cases for intake structures design with similar geometry.

During the model tests, the three selected remedial measures are investigated. Although, it will be possible to study some useful empirical relationships for different hydraulic or geometry parameters. We must be satisfied by only three remedial measures because the practical studies are also limited by deadline and budget.

The pump inlet disturbances contain a series of flow phenomena and hence, the careful should be taken into account during the intake structure design process. The physical model represents an additional cost during the design process. Nevertheless, these costs are significantly lower compared to the costs which arise in the case when troubleshooting appears during the operation of the pumping system. A rehabilitation of the pumping system during the operations can be costly and demanding. In addition, it reduces the availability of the pumping system, which consequently results in a greater financial loss [1] and [3].

\section{ACKNOWLEDGMENTS}

The study is the result of the years of cooperation between the Nuclear Power Plant Krško and the Kolektor Turboinštitut.

\section{NOMENCLATURES}

$D \quad$ suction bell diameter, [mm]

$B$ distance from the back wall to the pump, [m]

$C$ the distance between the inlet bell and floor, [m]

$d$ suction channel diameter, [mm]

$\varepsilon \quad$ relative friction ratio, [-]

$e_{d 1}$ systematic error of flow meter, [-]

$e_{d 2}$ random error of flow through the orifice plate, [-]

$e_{z 1} \quad$ systematic error of pressure transducer, [-]

$e_{z 2}$ random error of pressure transducer, [-]

$e_{Q}$ total measurement uncertainty of flow rate, [-]

$e_{Q d}$ measurement uncertainty of the flow rate over the dam, [-]

$e_{Q z}$ measurement uncertainty of the intake flow, [-]

$\mathrm{Fr}$ Froude number, [-]

$g$ gravity acceleration, $\left[\mathrm{m} / \mathrm{s}^{2}\right]$

$L$ length, [m]

$n$ angular velocity, [-]

$n q$ specific speed of the pump, [-]

$\rho$ density, $\left[\mathrm{kg} / \mathrm{m}^{3}\right]$

$v$ kinematic viscosity, $\left[\mathrm{m}^{2} / \mathrm{s}\right]$

Re Raynolds number, [-]

$S$ submergence, $[\mathrm{mm}]$

Scr critical submergence, $[\mathrm{mm}]$

$X$ pump inlet bay length, [m]

$Q$ flow rate, $\left[\mathrm{m}^{3} / \mathrm{s}\right]$

$V$ flow velocity, $[\mathrm{m} / \mathrm{s}]$

$V c$ cross-flow velocity, $[\mathrm{m} / \mathrm{s}]$

$V x$ pump bay velocity, [m/s]

$W$ pump bay entrance width, [m]

We Weber number, [-]

$u$ axial velocity, $[\mathrm{m} / \mathrm{s}]$

$\theta \quad$ vorticity angle, $\left[{ }^{\circ}\right]$

$Z_{1}$ distance from pump inlet bell centerline to diverging walls, $[\mathrm{m}]$

\section{REFERENCES}

[1] Florjančič, D. (2008). Trouble-shooting, Handbook for Centrifugal Pumps. Turboinštitut, Ljubljana.

[2] Gülich, J.F. (2013). Centrifugal Pumps. Springer-Verlag, Berlin.

[3] Hydraulic Institute (2001). Pump Life Cycle Costs, A Guide to LCC Analysis for Pumping System, Hydraulic Institute, Parsippany.

[4] ANSI/HI 9.8-2012 (2012). Rotodynamic Pumps for Pump Intake Design. American National Standard Institute, New Jersey. 
[5] Ansar, M. (1997). Experimental and Theoretical Studies of Pump Approach Flow Distributions at Water Intakes. Ph.D. thesis, The University of lowa, lowa City.

[6] Quick, M.C. (1970). Efficiency of air-entraining vortex at water intakes. Journal of the Hydraulics Division, American Society of Civil Engineers, vol. 96, no. 7, p. 1403-1415.

[7] Denny, D.F. (1956). An experimental study of air-entraining vortices in pump sumps. Proceedings of the Institution of Mechanical Engineers, vol. 170, no. 1, p. 106-125, DOI:10.1243/PIME_PROC_1956_170_019_02.

[8] Padmanabhan, M., Hecker, G.E. (1984). Scale effects in pump sump models. Journal of Hydraulic Engineering, vol. 110, no. 11, p. 1540-1556, Dol:10.1061/(ASCE)07339429(1984)110:11(1540).

[9] Anwar, H.O., Amimilett, M.B. (1980). Vortices at vertically inverted intake. Journal of Hydraulic Research, vol. 18, no. 2, p. 123-134, DOI:10.1080/00221688009499556.

[10] Tagamori, M., Ueda, H. (1991). An Experimental Study of Submerged Vortices and Flow Pattern in the Pump Sump. Japanese Society of Mechanical Engineering, Paper 91-0562, DOI:10.1299/kikaib.57.3641.

[11] Ansar, M., Nakato T. (2001). Experimental study of 3D pumpintake flows with and without cross flow. Journal of Hydraulic Engineering, vol. 127, no. 10, p. 825-834, D0l:10.1061/ (ASCE)0733-9429(2001)127:10(825).

[12] Hwang, K.S., Yang, C.H. (2003). Hydraulics model testing of circulating-water pump sump in Shen-Ho power plant, Research Report 293. Taiwan Hydraulics Laboratory, Tainan.
[13] Li, S., Lai, Y., Weber, L., Silva, J.M., Patel, V.C. (2004). Validation of a three-dimensional numerical model for waterpump intakes. Journal of Hydraulic Research, vol. 42, no. 3, p. 282-292, DOI:10.1080/00221686.2004.9728393.

[14] Tokyay, T.E., Constantinescu, S.G. (2006). Validation of a large-eddy simulation model to simulate flow in pump intakes of realistic geometry. Journal of Hydraulic Engineering, vol. 132, no. 12, p. 1303-1315, D0l:10.1061/(ASCE)07339429(2006)132:12(1303).

[15] Yuilin, W., Yong, L., Xiaoming, L. (2000). PIV Experiments on Flow in a Model Pump Suction Sump, Research Report, Thermal Engineering Department, Tsinghua University, Beijing.

[16] Škerlavaj, A., Vehar, F., Pavlin, R., Lipej, A. (2009). A hydraulic study of cooling water intake structure. Proceedings of the 3 rd IAHR International Meeting of the Workgroup on Cavitation and Dynamic Problems in Hydraulic Machinery and Systems, p. $143-154$.

[17] Munson, B.R., Okiishi, T.H., Huebsch, W.W., Rothmayer A.P. (2012). Fundamentals of Fluid Mechanics, Wiley, Hoboken.

[18] Franch, R.H. (1986). Open-Chanel Hydraulic. Mc Graw-Hill, Singapore.

[19] NEK (2017). Plant System and Operation, from http://www. nek.si, assessed on 2017-10-10.

[20] Pavlin, R., Žvab, G. (2016). Analyse of Pump Intake Conditions with Model Test: Analyse of Intake Structures with Model Test Procedure, Report no.: 3164-1-0, Krško. 